\title{
P02-14-8 Poster session
}

\section{The absorptive property of Paeoniflorin-6-O'-benzenesulfonate (CP-25) in peripheral blood mononuclear cells}

\author{
Chun Wang ${ }^{1,2,3}$, Min-Yi Zhao ${ }^{1,2,3}$, Jian Wang ${ }^{1,2,3}$, Yi-Jin Wu ${ }^{1,2,3}$, Wei Wei $i^{1,2,3}$ \\ ${ }^{1}$ Institute of Clinical Pharmacology, Anhui Medical University, China, ${ }^{2}$ Key Laboratory of Anti-inflammatory and \\ Immune Medicine, Ministry of Education, China, ${ }^{3}$ Collaborative Innovation Center of Anti-inflammatory and Immune \\ Medicine in Anhui Province, China
}

Objective Paeoniflorin-6'O-benzene sulfonate (CP-25) is a newly derivative of Paeoniflorin (Pae). We have previously reported CP-25 shows better anti-inflammatory activity and higher bioavailability than that of Pae. This study was mainly performed to investigate absorptive behavior and mechanism of CP-25 in PBMCs both in vivo and vitro.

Methods Peripheral blood mononuclear cells (PBMCs) were collected from normal rats and stimulated by TNF-a for imitating the inflammatory response in vitro. A speci\&\#64257;c and sensitive ultra-performance liquid chromatography tandem mass spectrometry (UPLC-MS/MS) assay for rapid determination of CP-25 and Pae in the rats' PBMCs was developed and validated for the first time.

Results CP-25 had a maximum intracellular level in the rats' PBMCs at 120 min after incubation in vitro. Likewise, CP-25 showed an maximum intracellular level in PBMCs at $3 \mathrm{~h}$ after the oral signal administration. Interestingly, the intracellular levels of CP-25 were higher than Pae both in vivo and vitro. Moreover, TNF-a pre-stimulated PBMCs showed an increase in the level of P-gp expression, and an reduced absorption of CP-25, which partially inhibited by P-gp inhibitors, including GF120918, LY335979 and verapamil. In vivo studies, the P-gp levels in PBMCs of rats with adjuvant arthritis (AA) were significantly higher than that of normal rats, and we noticed the level of CP-25 in PBMCs of AA rats were significantl higher than that of norma rats.

Conclusion Absorption of CP-25 in PBMCs is better than Pae both in vitro and vivo. Inflammatroy response induced increase in the P-gp expression limited the permeable absorption of CP-25 at intracelluar levels.

Key words: CP-25; Paeoniflorin; Peripheral blood mononuclear cells; P-glycoprotein

Acknowledgments

This work was supported by the National Natural Science Foundation of China (No.81330081, No. 81302845).

Corresponding author: Prof. Wei Wei; Email: wwei@ahmu.edu.cn;

Institute of Clinical Pharmacology, Anhui Medical University, 230032, Hefei, China.

The presentation of first author:

Chun Wang received his degrees of M.S. and Ph.D. from Institute of Clinical Pharmacology of Anhui Medical University. The main research direction of Chun Wang is the roles of $\mathrm{ABC}$ transporters \& organic anion transporters in the cellular pharmacokinetics of anti-inflammatory drugs at auto-immune diseases and new immunomodulatory drugs development. 